Materials science

\section{Novel model for cavity lattices}

\section{Robert W. Cahn}

Microscopic cavities, either empty (voids) or gas-filled (bubbles), created in crystalline solids by irradiation or ion implantation, are apt to form lattices that mimic the host lattice but are coarser by a factor of $70-120$. These entities are termed void or bubble lattices; cavity lattice covers both categories. Void lattices were originally discovered in molybdenum by Evans' at the Atomic Energy Research Establishment at Harwell, United Kingdom, where much of the subsequent work has been done. Cavity lattices have been observed in metals with various structures, alumina and alkaline earth halides. Johnson et al. ${ }^{2}$, also working at Harwell, on page 316 of this issue, propose a radically new mechanism, an inter-cavity repulsion caused by 'dislocation punching'. This joins several other mechanisms that have been proposed (reviewed in refs 3,4 ) to account for the formation of cavity lattices.

Cavity lattices in cubic metals are always in 'parallel orientation' and fully developed in three dimensions; in hexagonal-close-packed (hcp) metals, however, voids aggregate in sheets parallel to the basal plane, without internal ordering in the sheets. Most current theoretical models address the formation of paralleloriented cavity lattices. It was recognized soon after Evans's original discovery that an elastic intėraction between voids or bubbles arises from the anisotropy of the elastic constants; there are both attractive and repulsive components so that an optimum separation between cavities results. But elastically isotropic metals like tungsten also form voids, a difficulty that is overcome if the spherical voids are allowed to distort and develop flat faces. Although the stability of a lattice, once formed, can certainly be explained in terms of this rigorously developed static model, it is still uncertain whether the energy minimum at the preferred cavity separation is deep enough to permit lattice formation in the first place on the basis of this model (J.H. Evans, personal communication).

\section{Dynamic theories}

More recently, dynamic theories have been developed on the basis of anisotropic diffusion of self-interstitial atoms (SIAs); in the original form, constrained onedimensional diffusion or dynamic replacement sequences were assumed. This kind of mechanism can be effective because unaligned voids will receive a greater flux of SIAs than those which are already aligned, because the latter will be locally shadowed by cavities already in the lattice. Cavities migrate in a crystalline solid by a mechanism involving surface selfdiffusion along the cavity wall - the more rapidly, the smaller the cavity - and the flux of SIAs accelerates such migration. Local shadowing acts as a feedback control mechanism tending to perfect a lattice. The most recent variant of this approach, originally proposed in 1972, is by Woo and Frank ${ }^{5}$. This kind of model has also been embraced by those seeking to interpret cavity lattice formation in non-metals ${ }^{4}$.

An alternative form of SIA model, recently advanced by Evans ${ }^{6}$, involves two-dimensional diffusion of SIAs confined within a crystal lattice plane. Computer simulation has shown that this kind of motion, for which there is sound theoretical basis, can by degrees convert a random cavity array into an ordered one. This is the only model up to now that can explain the planar ordering of cavities in hcp metals. Unlike the elastic anisotropy approach, however, this model is less clear about the magnitude of the interaction holding the lattice in place once it is formed. The older model interprets stability, the newer ones concentrate on initial formation.

The latest Harwell model is intended to interpret a novel set of observations, also reported in the paper of Johnson et al. in this issue ${ }^{2}$. The authors injected helium into copper (a venerable technique, first used in 1960 by Barnes and Mazey - the latter is one of the authors of the new paper - in the demonstration of bubble mobility in a solid metal). Helium bubbles about $2 \mathrm{~nm}$ in diameter are created at a concentration of about $10^{25} \mathrm{~m}^{-3}-$ an unusually high concentration - and form a bubble lattice, accurate enough to give satellite maxima in electron diffraction. What is novel about this lattice is that parts of it are not in parallel orientation: two types of domain are present sharing a common $\{111\}$ plane with the paralleloriented domains, but are mutually rotated about the perpendicular $<111>$ direction. This is the first firm observation of cavity lattice domains in non-parallel orientation.

The authors go on to point out that small noble-gas bubbles formed by ion implantation are always grossly overpressurized, in the sense that the surface tension alone does not suffice to contain the gas pressure. The crystal surrounding each bubble is consequently under great stress. Such bubbles can grow by absorbing vacancies created by radiation damage caused by the implanting beam, but when these are exhausted, the only further way a bubble can grow (and thus reduce its overpressure) is either by merging with another bubble - not feasible in a lattice with repulsion between bubbles that approach each other too closely - or by plastically deforming the stressed crystal matrix surrounding the bubble.

One mechanism of plastic deformation that has been well documented ${ }^{7.8}$ is dislocation punching: a succession of mobile dislocation rings, of the same diameter as the bubble, is dispatched from the bubble along a 'glide cylinder', the axis of which coincides with the Burgers vector, $<011>$, away from the surface of the bubble, providing more room for it.

\section{Mutual repulsion}

Thus, neighbouring helium bubbles disposed so that a $<011>$ vector joins them, experience a mutual repulsion from the dislocation rings which each bubble emits towards the other, as has indeed been previously recognized". (Dislocations of the same sign necessarily repel each other and their sources.) This mutual repulsion may suffice to overcome the elastic attraction which holds the bubble lattice together, and the authors demonstrate $^{2}$ that this process will convert the parallel orientation into one or other of the two observed alternative orientations, in both of which the bubble separations parallel to $\langle 011\rangle$ are increased by a factor of at least 3 . The experiments reported in this issue involve particularly high helium concentrations and close bubble spacings (about $6 \mathrm{~nm}$ ) so that bubble repulsions should be unusually strong.

Studies of cavity lattices such as this have a bearing on the ordering, on lattices, of other structural entities. Khachaturyan and Airapetyan ${ }^{\text {i) }}$ have demonstrated that elastic interactions can account for the formation of precipitate lattices. In principle, precipitates could also stress the surrounding matrix crystal lattice if their unconstrained atomic volumes exceed that of the matrix lattice, and conceivably dislocation punching could also be involved in the formation of this kind of macrolattice.

1. Evans, J.H. Nature 229, 403-404 (1971).

2. Johnson, P.B., Malcolm. A.L. \& Mazey, D.J. Nature 328, 316-318 (1987).

Krishan. K. Radiat. Effects 66, 121 - 155 (1982)

4. Johnson, E. \& Chadderton. L.T. Radiat. Effects 79, 183 233 (1983).

5. Woo, C.H. \& Frank, W. Mater. Sci. Forum 15-18, 875 $880(1987)$

6. Evans, J.H. Mater. Sci. Forum 15-18, 869-874 (1987),

7. Barnes, R.S. \& Mazey, D.J. Acta metall. 11, 281-286 (1983).

8. Evans, J.H. J. nucl. Mater. 76 \& 77, 125-132 (1978).

9. Dubinko, V.I., Slezov, V.V.. Tur., A.V. \& Yanovsky, V.V. Radiat. Effects 100, 85-104 (1986).

10. Khachaturyan, A.F. \& Airapetyan, V.M. Phys. Status Solidi. A 26, 61-70 (1974)

Robert W. Cahn is in the Department of Materials Science and Metallurgy, Cambridge University, Cambridge CB2 $3 Q Z$, $U K$. 